\title{
Hyperkähler Manifolds and Nonlinear Supermultiplets
}

\author{
A. Karlhede ${ }^{1, \star}$, U. Lindström ${ }^{2, \star \star}$, and M. Roček ${ }^{2, \star \star \star}$ \\ ${ }^{1}$ Institute of Theoretical Physics, University of Stockholm, Vanadisvägen 9, \\ S-11346 Stockholm, Sweden \\ ${ }^{2}$ Institute for Theoretical Physics, State University of New York, Stony Brook, \\ NY 11794, USA
}

\begin{abstract}
We present a new construction of hyperkähler metrics that derives from the 3-dimensional $N=4$ nonlinear supermultiplet. Further, we give a detailed description of the nonlinear multiplet in $N=2$ and 4 superspace.
\end{abstract}

\section{Introduction}

In this paper we present a new construction of hyperkähler metrics. We use the method introduced in [1] and discussed extensively in [2]: We construct $N=4$ supersymmetric nonlinear $\sigma$-models in terms of an off-shell multiplet, here the nonlinear multiplet, and then find a dual transformation to the formulation in terms of $N=2$ chiral superfields. This yields the Kähler potential, and hence the metric, explicitly. We also discuss the superfield formulation of the nonlinear multiplet in $N=2$ and 4 extended superspace.

In Sect. II, we give the construction without any reference to supersymmetry. In Sect. III, we discuss the nonlinear multiplet, first in $N=4$, and then reduced to $N=2$ superspace. In Sect. IV, we derive the construction of Sect. II. We use the notation of [2] throughout.

\section{Construction of New Hyperkähler Metrics}

In this section, we follow the discussion of the Legendre transform construction of hyperkähler metrics of [2, Sect. 2A], as closely as possible. We start with a $3 n$ real dimensional space $\mathbf{E} \equiv\left(\mathbf{S}^{3}\right)^{n}$ embedded in $\overline{\mathbf{E}} \equiv\left(\mathbf{C}^{2}\right)^{n}$. The coordinates $x^{i}, z^{i}, \bar{z}^{i}$ $(i=1, \ldots, n)$ on $\mathbf{E}$ are defined in terms of the coordinates $w^{i}, v^{i}$ on $\overline{\mathbf{E}}$ by

$$
z^{i}=\frac{\bar{w}^{i}}{v^{i}}, \quad \exp \left(i x^{i}\right)=\frac{v^{i}}{\bar{v}^{i}}
$$

\footnotetext{
* Supported by the Swedish Natural Science Research Council

$\star \star$ On leave of absence from ITP, University of Stockholm

$\star \star \star$ Supported in part by NSF Grant No. PHY 85-07627
} 
We consider a real function $F: \mathbf{E} \rightarrow \mathbf{R}$, i.e., $F\left(x^{i}, z^{i}, \bar{z}^{i}\right)$, that satisfies the system of linear differential equations,

$$
\begin{gathered}
F_{x^{i} x^{j}}+\exp \left(i\left(x^{i}-x^{j}\right)\right) F_{z^{j} \bar{z}^{i}}+z^{i} \bar{z}^{j} F_{z^{i} \bar{z}^{j}}+i\left[\bar{z}^{j} F_{x^{i} \bar{z}^{j}}-z^{i} F_{x^{j} z^{i}}\right]=0 \quad \text { (no sum), } \\
\exp \left(i\left(x^{i}-x^{j}\right)\right)\left(z^{j} F_{z^{j} \bar{z}^{i}}+i F_{x^{j} \bar{z}^{i}}\right)=z^{i} F_{z^{i} \bar{z}^{j}}+i F_{x^{i} \bar{z}^{j}} \quad \text { (no sum). }
\end{gathered}
$$

For each $i,(2.2 \mathrm{a})$ is just the Laplace equation on the three sphere. A characterization of $F$, equivalent to (2.2), is as a contour integral in an auxiliary variable $\zeta$ :

$$
F(x, z, \bar{z})=\frac{1}{2 \pi i} \oint\left(\zeta^{-2}\right) d \zeta G\left(\eta^{i}, \zeta\right)
$$

where

$$
\eta^{i}=\frac{\bar{z}^{i}+\zeta \exp \left(i x^{i}\right)}{z^{i \zeta} \exp \left(i x^{i}\right)-1}
$$

The Kähler potential is given by a nonlinear analog of a Legendre transform:

$$
K(u, \bar{u}, z, \bar{z})=F(x, z, \bar{z})+\sum\left(1+z^{i} \bar{z}^{i}\right)\left[u^{i} \exp \left(-i x^{i}\right)+\bar{u}^{i} \exp \left(i x^{i}\right)\right],
$$

where $x^{i}$ is a function of $z^{i}, \bar{z}^{i}, u^{i}$, and $\bar{u}^{i}$ determined by

$$
K_{x^{i}}=0=F_{x^{i}}+i\left(1+z^{i} \bar{z}^{i}\right)\left[\bar{u}^{i} \exp \left(i x^{i}\right)-u^{i} \exp \left(-i x^{i}\right)\right] \text { (no sum). }
$$

The metric of the manifold is computed in the standard way from the Kähler potential (2.5),

$$
d s^{2}=2\left(K_{u_{\bar{u}} j} d u^{i} \otimes d \bar{u}^{j}+K_{z^{i} \bar{u}^{j}} d z^{i} \otimes d \bar{u}^{j}+K_{u^{i} \bar{z}^{j}} d u^{i} \otimes d \bar{z}^{j}+K_{z^{i} \bar{z}^{j}} d z^{i} \otimes d \bar{z}^{j}\right) .
$$

Note that, in contrast with the Legendre transform construction $[1,2]$, the metric (2.7) does not have any obvious isometries. However, in these coordinates, the Kähler potential obeys the constraint $K_{u^{i}} K_{\bar{u}^{i}}=\left(1+z^{i} \bar{z}^{i}\right)^{2}$ for every $i=1, \ldots, n$. We do not know an invariant characterization of this constraint.

After the nonlinear transform (2.5), Eqs. (2.2) imply

$$
\left(K_{u^{j} \bar{u}^{i}}\right)^{-1}=K_{z^{i} \bar{z}^{j}}-K_{z^{i} \bar{u}^{k}}\left(K_{u^{k} \bar{u}^{m}}\right)^{-1} K_{u^{m} \bar{z}^{j}}
$$

which is Eq. (5.31) of [1] and imply the Monge-Ampère equation.

We observe that $\eta^{i}(x, z, \bar{z})$ can just as well be written in terms of the coordinates $w, v$ on $\overline{\mathbf{E}}$ :

$$
\eta^{i}=\frac{w^{i}+\zeta v^{i}}{\zeta \bar{w}^{i}-\bar{v}^{i}},
$$

and that consequently, $F\left(w^{i}, \bar{w}^{i}, v^{i}, \bar{v}^{i}\right)$ defined by (2.3) satisfies the linear equations on $\overline{\mathbf{E}}$ [equivalent to (2.2)],

$$
\begin{gathered}
F_{w^{i} \bar{w}^{j}}+F_{v^{i} \bar{v}^{j}}=0 \\
F_{w^{i} v^{j}}=F_{v^{i} w^{j}}
\end{gathered}
$$


For comparison, the Legendre transform construction of $[1,2]$ corresponds to the solution of the Eq. (2.10) written as (2.3) with

$$
\eta_{L}^{i}=w^{i}-\zeta\left(v^{i}+\bar{v}^{i}\right)+\zeta^{2} \bar{w}^{i}
$$

which corresponds to embedding $(\mathbf{R} \otimes \mathbf{C})^{n}$ rather than $\mathbf{E}=\left(\mathbf{S}^{3}\right)^{n}$ in $\overline{\mathbf{E}}$. In the Legendre transform construction, $(2.10 \mathrm{~b})$ can be thought of as a coordinate choice. In $[1,2]$, this condition was not imposed; it leads to significant simplifications, in particular for the holomorphic two form that generates the quaternionic structure, which becomes $\omega^{+}=4 d u^{i} \wedge d z^{i}$, cf. Eq. (2.8) of [2]; see (2.14) below. Clearly, we can combine the Legendre transform construction with the nonlinear one presented here by considering a function $G\left(\eta^{i}, \eta^{j}, \zeta\right)$, with $i=1, \ldots, k$, and $j=(k+1), \ldots, n$.

The Eqs. (2.6) cannot in general be solved explicitly for $x^{i}$. As in the Legendre transform construction, we can compute the line element explicitly in nonholomorphic coordinates. We use the original coordinates $x^{i}, z^{i}$, and $\bar{z}^{i}$, and $n$ additional real coordinates, e.g.,

$$
y^{i}=\left(1+z^{i} \bar{z}^{i}\right)\left[\bar{u}^{i} \exp \left(i x^{i}\right)+u^{i} \exp \left(-i x^{i}\right)\right] \quad \text { (no sum). }
$$

The line element in these coordinates is (2.7) with

$$
\begin{aligned}
& K_{u^{i \bar{u} j}}=-\left(\varrho^{i} \varrho^{j}\right)^{-1} \exp \left(i\left(x^{j}-x^{i}\right)\right) A_{i j}, \quad A_{i j} \equiv\left(F_{x^{i} x^{j}}-\delta_{i j} y^{i}\right)^{-1}, \\
& K_{u^{i} \bar{z}}=\exp \left(-i x^{i}\right)\left(\delta_{i j} z^{i}+i A_{i k} B_{k j}\right), \quad B_{k j} \equiv F_{x^{k} \bar{z}^{j}}+\delta_{k j} \varrho^{j} z^{j} F_{x^{j}}, \\
& K_{z^{i} \bar{z}^{j}}=F_{z^{i} \bar{z} j}+\delta_{i j} \varrho^{i} y^{i}-\bar{B}_{i m} A_{m k} B_{k j}, \quad \bar{B}_{i m}=F_{z^{i} x^{m}}+\delta_{i m} \varrho^{i} \bar{z}^{i} F_{x^{i}}, \\
& \varrho^{i}=\left(1+z^{i} \bar{z}^{i}\right)^{-1}, \\
& d u^{i}=\frac{1}{2} \varrho^{i} \exp \left(i x^{i}\right)\left(\begin{array}{c}
d y^{i}-i\left(F_{x^{i} x^{j}} d x^{j}+F_{x^{i} z_{j}} d z^{j}+F_{x^{i} z_{j}} d \bar{z}^{j}\right) \\
+\left(y^{i}-F_{x^{i}}\right)\left(i d x^{i}-\varrho^{i}\left(z^{i} d \bar{z}^{i}+\bar{z}^{i} d z^{i}\right)\right)
\end{array}\right) .
\end{aligned}
$$

We can also explicitly construct the quaternionic structure of the hyperkähler manifold. In the notation of [2], we have

$$
\begin{aligned}
\omega^{1} & =2 i\left(K_{u^{i} \bar{u} j} d u^{i} \wedge d \bar{u}^{j}+K_{z^{i} \bar{u} j} d z^{i} \wedge d \bar{u}^{j}+K_{u^{i} \bar{z} j} d u^{i} \wedge d \bar{z}^{j}+K_{z^{i} \bar{z}_{j} j} d z^{i} \wedge d \bar{z}^{j}\right), \\
\omega^{+} & =4 d u^{i} \wedge d z^{i},
\end{aligned}
$$

which is precisely (2.8) of [2] with the simplification noted above.

We close this section by noting that flat space is generated by the trivial function $F(x, z, \bar{z})=0$. Though it is easy to find many examples locally, we have not analyzed their global properties, and, in contrast to the Legendre transform construction, have not found a useful relation to the symplectic quotient construction of hyperkähler metrics [1,2] (which would simplify the global analysis).

\section{Nonlinear Multiplet}

In this section, we describe the nonlinear multiplet [3] in $N=4$ and 2 superspace. In the next section, we use the multiplet to derive the construction of Sect. II. We use the notation of [2]; see also [4]. 
The nonlinear multiplet was introduced in the context of local conformal supersymmetry [3]. Here we only consider its description in global (rigid) superspace. In $N=4$ superspace, the multiplet is described by a matrix superfield $\Phi_{m a}$ that is an isospinor with respect to $t w o S U(2)$ groups: $m=1,2$ and $a=1,2$. It obeys a hermiticity relation

$$
\Phi_{m a}=\varepsilon_{m n} \varepsilon_{a b} \bar{\Phi}^{n b},
$$

a nonlinear constraint $\operatorname{det}\left(\Phi_{m a}\right)=1$, and a differential constraint

$$
\varepsilon^{m n} \Phi_{m(a} D_{\beta b} \Phi_{n c)}=0, \quad \varepsilon_{m n} \bar{\Phi}^{m(a} \bar{D}_{\beta}{ }^{b} \bar{\Phi}^{n c)}=0,
$$

where $D_{\alpha a}, \bar{D}_{\alpha}{ }^{a}$ are complex $N=4$ spinor-isospinor derivatives (see, e.g., [5]), with spacetime spinor index $\alpha=+,-$. The constraints imply that for any $\zeta$,

$$
\eta(\zeta)=\frac{\Phi_{11}+\Phi_{12} \zeta}{\bar{\Phi}^{11} \zeta-\bar{\Phi}^{12}}=\frac{\Phi_{11}+\Phi_{12} \zeta}{\Phi_{21}+\Phi_{22} \zeta}
$$

obeys

$$
\begin{gathered}
\nabla_{\alpha}(\zeta) \eta(\zeta)=\bar{\nabla}_{\alpha}(\zeta) \eta(\zeta)=0, \\
\nabla_{\alpha}(\zeta) \equiv D_{\alpha 1}+\zeta D_{\alpha 2}, \quad \bar{\nabla}_{\alpha}(\zeta) \equiv \bar{D}_{\alpha 2}-\zeta \bar{D}_{\alpha 1} .
\end{gathered}
$$

We can thus write down a general $N=4$ supersymmetric action for $n$ nonlinear multiplets $\Phi_{m a}^{i}, i=1, \ldots, n$ :

$$
\begin{gathered}
S=\int d^{3} x \int d \zeta \Delta^{2} \bar{\Delta}^{2} G\left(\eta^{i}, \zeta\right), \\
\Delta_{\alpha}(\zeta) \equiv D_{\alpha 2}-\zeta^{-1} D_{\alpha 1}, \quad \bar{\Delta}_{\alpha}(\zeta) \equiv \bar{D}_{\alpha 1}+\zeta^{-1} D_{\alpha 2} .
\end{gathered}
$$

Unfortunately, we do not have an unconstrained formulation of the nonlinear multiplet, and consequently, we are unable to derive superfield equations from the action (3.5).

We now give a description of the nonlinear multiplet in $N=2$ superspace. We choose $D_{\alpha 1}$ and $\bar{D}_{\alpha}{ }^{1}$ as our $N=2$ derivatives, and generate extra supersymmetries with the remaining spinor derivatives (see [5] for a description of this procedure). Then we define the following $N=2$ superfields

$$
\chi=\frac{\bar{\Phi}^{11}}{\Phi_{12}}\left|, \quad \exp (i X)=\frac{\Phi_{12}}{\bar{\Phi}^{12}}\right|,
$$

where $\mid$ denotes a projection to the subspace independent of the second spinor supercoordinate. The reality constraint (3.1) implies that $X$ is real, and the differential constraint (3.2) implies the $N=2$ constraints

$$
\begin{gathered}
\bar{D}_{\alpha} \chi=0, \\
\bar{D}^{2}\left[(1+\chi \bar{\chi}) e^{i X}\right]=0,
\end{gathered}
$$

as well as the extra supersymmetry transformations

$$
\begin{gathered}
\delta \chi=-\frac{1}{2} \bar{D}^{2}\left[\bar{\Lambda}(1+\chi \bar{\chi}) e^{-i X}\right], \\
\delta X=i(D \Lambda) D\left(\chi e^{i X}\right)+\text { c.c. },
\end{gathered}
$$


where the parameter of the transformations $\Lambda$ is a spatially constant chiral superfield constrained by $\bar{D} \Lambda=D^{2} \Lambda=\partial_{a} \Lambda=0$. The action (3.5) reduces to an $N=2$ superspace integral,

$$
S=\int d^{3} x D^{2} \bar{D}^{2} F\left(X^{i}, \chi^{i}, \bar{\chi}^{i}\right) .
$$

This is invariant under the transformations (3.8) when $F(X, \chi, \bar{\chi})$ is given by

$$
\begin{gathered}
F\left(X^{i}, \chi^{i}, \bar{\chi}^{i}\right)=\frac{1}{16} \oint\left(\zeta^{-2}\right) d \zeta G\left(\eta^{i}, \zeta\right), \\
\eta^{i}(\zeta)=\frac{\bar{\chi}^{i}+\zeta \exp \left(i X^{i}\right)}{\chi^{i \zeta} \exp \left(i X^{i}\right)-1} .
\end{gathered}
$$

Identifying the chiral superfields $\chi^{i}$ with the complex coordinates $z^{i}$, we see that this is just the form (2.3), and implies that $F$ satisfies the system of linear equations (2.2).

\section{Origin of the New Construction of Hyperkähler Metrics}

With the tools assembled in the previous section, it is simple to derive the construction of Sect. II. We start with the $N=2$ superspace action (3.9); we relax the constraints (3.7b), and impose them in the action by introducing $n$ chiral Lagrange multipliers $\Phi^{i}$ (cf. [1, 2]):

$$
S^{1}=\int d^{3} x D^{2} \bar{D}^{2}\left[F\left(\Psi^{i}, \chi^{i}, \bar{\chi}^{i}\right)+\sum\left(1+\chi^{i} \bar{\chi}^{i}\right)\left(\Phi^{i} \exp \left(-i \Psi^{i}\right)+\bar{\Phi}^{i} \exp \left(i \Psi^{i}\right)\right)\right]
$$

In this action, $X^{i}$ has been replaced by the unconstrained superfield $\Psi^{i}$. Extremizing the action with respect to $\Psi^{i}$, we find

$$
F_{\Psi^{i}}+i\left(1+\chi^{i} \bar{\chi}^{i}\right)\left[\bar{\Phi}^{i} \exp \left(i \Psi^{i}\right)-\Phi^{i} \exp \left(-i \Psi^{i}\right)\right]=0 \quad \text { (no sum), }
$$

which is to be solved for $\Psi^{i}(\chi, \bar{\chi}, \Phi, \bar{\Phi})$. This leads to a Kähler potential

$$
K(\chi, \bar{\chi}, \Phi, \bar{\Phi})=F\left(\Psi^{i}, \chi^{i}, \bar{\chi}^{i}\right)+\sum\left(1+\chi^{i} \bar{\chi}^{i}\right)\left(\Phi^{i} \exp \left(-i \Psi^{i}\right)+\bar{\Phi}^{i} \exp \left(i \Psi^{i}\right)\right) ;
$$

identifying the superfields $\Psi, \Phi$ with the coordinates $x, z$, we find $(2.5,6)$. The quaternionic structure (2.14) follows from the nonmanifest supersymmetry (3.8) extended to (4.3); eliminating $\Psi$ by its variational equation (4.2) we find:

$$
\delta \chi^{i}=-\frac{1}{2} \bar{D}^{2}\left(\bar{\Lambda} K_{\Phi^{i}}\right), \quad \delta \Phi^{i}=\frac{1}{2} \bar{D}^{2}\left(\bar{\Lambda} K_{\chi^{i}}\right) .
$$

This is identical to Eq. (5.32b) of [1] after the simplification discussed above. This concludes the derivation of the construction of Sect. II.

The obvious open problems that remain are: (1) To find a classification of the metrics that can be constructed using the nonlinear transform. The corresponding classification is known for the Legendre transform: All $4 n$-dimensional hyperkähler metrics with at least $n$ commuting triholomorphic isometries can be constructed $[6,2]$. (2) To make an analysis of the global behavior of metrics that can be constructed by our new method.

Acknowledgements. We would like to thank the ITP's at Stony Brook and Stockholm for mutual hospitality while this work was in progress. We would also like to thank J. Labastida, E. Velasco, and P. Wills for checking some of the calculations in Sect. III. 


\section{References}

1. Lindström, U., Roček, M.: Scalar tensor duality and $N=1,2$ nonlinear $\sigma$-models. Nucl. Phys. B 222, 285 (1983)

2. Hitchin, N.J., Karlhede, A., Lindström, U., Roček, M.: Hyperkähler metrics and supersymmetry. Commun. Math. Phys. 108, 535-589 (1987)

3. de Wit, B., van Holten, J.W., Van Proeyen, A.: Structure of $N=2$ supergravity. Nucl. Phys. B 184, 77 (1981)

4. Gates, S.J., Grisaru, M.T., Roček, M., Siegel, W.: Superspace, or one thousand and one lectures in supersymmetry, Chap. 2. Reading, MA: Benjamin/Cummings 1983

5. Gates, S.J., Hull, C.M., Roček, M.: Twisted multiplets and new supersymmetric nonlinear $\sigma$ models. Nucl. Phys. B 248, 157 (1984)

6. Howe, P.S., Karlhede, A., Lindström, U., Roček, M.: The geometry of duality. Phys. Lett. 168 B, 89 (1986)

Communicated by L. Alvarez-Gaumé

Received November 1, 1986 